Denniston, J. C., Savastano, H. I., Blaisdell, A. P., \& Miller, R. R. (2003). Cue competition as a retrieval deficit. Learning and Motivation, 34(1): 1-31. (Feb 2003) Published by Elsevier (ISSN: 1095-9122). DOI:10.1016/S00239690(02)00505-2

\title{
Cue competition as a retrieval deficit
}

\author{
James C. Denniston, Hernan I. Savastano, Aaron P. Blaisdell, and Ralph R. Miller
}

\begin{abstract}
Four experiments using rats as subjects investigated the claim of Williams (1996) that cue competition results from an associative acquisition deficit, rather than a performance deficit. In Experiment 1, extinction of an overshadowing stimulus following overshadowing treatment increased responding to the overshadowed stimulus, thereby replicating prior observations with new parameters. In Experiment 2, an overshadowed stimulus failed to support second-order conditioning unless the overshadowing stimulus received prior extinction treatment. Experiment 3 replicated the recovery from overshadowing effect seen in Experiment 1 using a sensory preconditioning procedure. Most important, in Experiment 4 an overshadowed stimulus failed to block conditioned responding to a novel CS, but blocking by the overshadowed cue was observed following posttraining extinction of the overshadowing stimulus. These results, as well as those of Williams, are discussed in terms of traditional and more recent acquisition-focused models as well as an extension of the comparator hypothesis (Denniston, Savastano, \& Miller, 2001).
\end{abstract}




\section{CUE COMPETITION AS A RETRIEVAL DEFICIT}

Students of associative learning have long been interested in the conditions under which conditioned stimuli (CSs) trained together will compete with one another for behavioral control. One example of cue competition is the so-called "blocking" effect (Kamin, 1968). In a typical blocking experiment, a CS (A) is paired with an unconditioned stimulus (US) in Phase 1 (i.e., $A \rightarrow U S$ ); then in Phase 2, CS A and another stimulus (CS X) are paired with the US (i.e., $A X \rightarrow U S)$. Blocking is evidenced by reduced behavioral control by $C S X$ relative to subjects that received Phase 1 training with an irrelevant stimulus (i.e., $B \rightarrow U S$ ). Most contemporary theories of learning explain blocking and other forms of cue competition as a deficit in acquiring an X-US association. For example, Rescorla and Wagner (1972) explained blocking as a result of CS A accruing much of the associative value supportable by the US during Phase 1 . This strong AUS association leaves less potential associative strength to the US available for assignment during Phase 2 than if both $A$ and $X$ were novel at the time of Phase 2 training.

The comparator hypothesis, a rule for the expression of associations, provides an alternative account of the blocking phenomenon (e.g., Miller \& Matzel, 1988; Miller \& Schachtman, 1985). The comparator hypothesis assumes that associations are formed based upon a simple associative acquisition mechanism that is entirely dependent upon spatio-temporal contiguity (e.g., Bush \& Mostellar, 1955) and the salience of the associates. It treats the weak conditioned responding observed in blocking as a performance deficit originating at the time of testing rather than an acquisition deficit occurring at the time of associative acquisition (i.e., Phase 2).

According to the comparator hypothesis, at least three associations are formed in the course of blocking training (see Fig. 1). The first association is between the target CS (X) and the US (Link 1). The second association is between the target CS (X) and the blocking CS (A), which is the so-called comparator stimulus for CS X (Link 2). The third association is between the blocking CS (A) and the US (Link 3). At test, conditioned responding to CS X is assumed to reflect a comparison of the US representations directly and indirectly activated by the target CS (X). The strength of the directly activated US representation reflects the absolute strength of the target CS-US association (Link 1), whereas the strength of the indirectly activated US representation is determined by the product of the strengths of Links 2 and 3 . Through the comparator process, excitatory behavioral control by the target CS is presumed to increase (and inhibitory behavioral control to decrease) as the strength of the directly activated US representation increases relative to the strength of the indirectly activated US representation. Conversely, excitatory behavioral control is presumed to decrease (and inhibitory behavioral control to increase) as the strength of the directly activated US representation decreases relative to the strength of the indirectly activated US representation. 


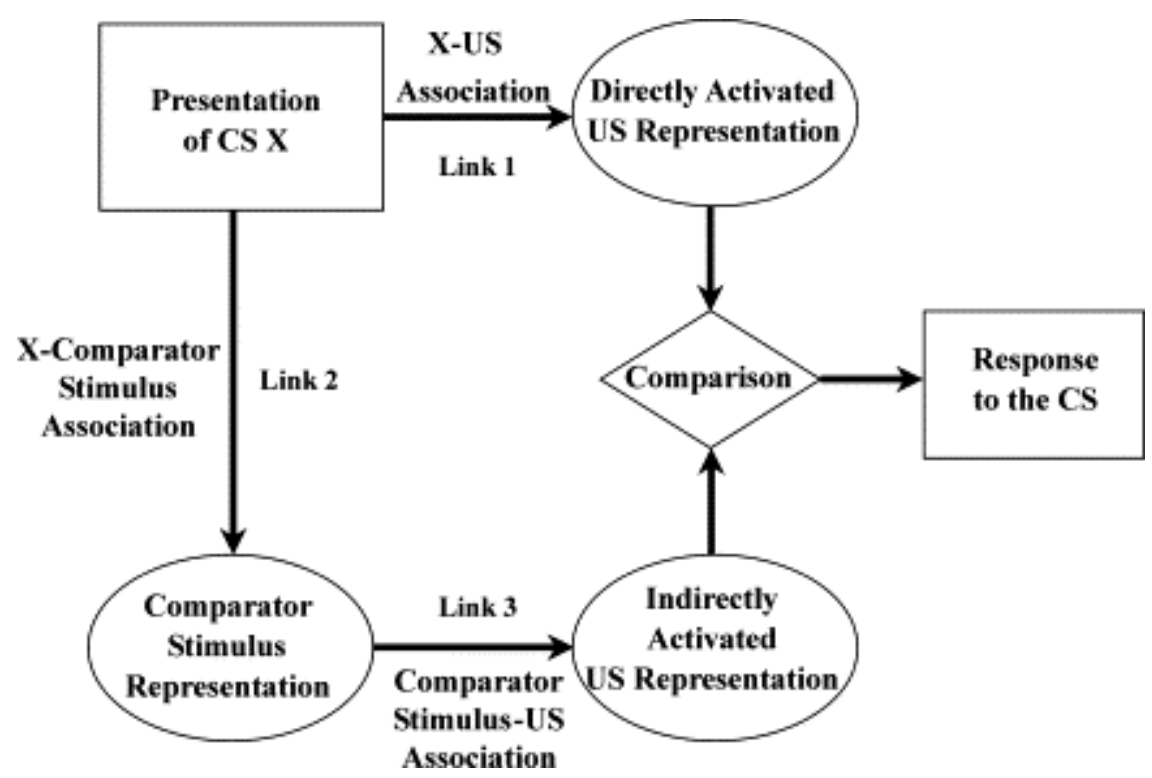

Fig. 1. The original comparator hypothesis (Miller \& Matzel, 1988; Miller \& Schachtman, 1985). CS, conditioned stimulus; US, unconditioned stimulus. Conditioned responding to CS X is directly related to the magnitude of the US representation that is directly activated by CS X and is inversely related to the magnitude of the US representation that is indirectly activated by CS X (i.e., mediated by CS X's comparator stimulus through conjoint action of the X-comparator stimulus and comparator stimulus-US associations).

Applied to blocking, the failure to observe responding to the blocked stimulus $X$ results from strong $\mathrm{X}-\mathrm{A}$ and $\mathrm{A}-\mathrm{US}$ associations preventing expression of the acquired $\mathrm{X}-\mathrm{US}$ association at the time of testing, rather than a failure to acquire the $X-U S$ association at the time of training. That is, in a conventional blocking group the product of the strengths of Links 2 and 3 will be greater than that in controls which lack prior A-US training and hence have a weaker $A \rightarrow U S$ association (Link 3). At test, the strength of the indirectly activated US representation will be stronger in the blocking group relative to the control group, thereby resulting in attenuated conditioned responding to CS X despite equivalent X-US associations (Link 1 ) in the two groups.

Recently, some researchers have questioned the performance-deficit account of cue competition effects provided by the comparator hypothesis. For example, Williams (1996) examined the potential of a blocked stimulus to subsequently serve as a blocking stimulus for a novel CS. That is, following blocking training ( $A \rightarrow U S$ in Phase 1 and $A X \rightarrow U S$ in Phase 2), Williams presented subjects with $X Y \rightarrow U S$ pairings (in Phase 3 ) and then examined responding to CS Y. According to the Rescorla-Wagner (1972) model, CS X, which presumably possessed little associative strength after Phase 2, should not have blocked conditioning to CS Y. In contrast, the comparator hypothesis claims that $\mathrm{CS} X$ became associated with the US during Phase 2, but that strong $\mathrm{X}-\mathrm{A}$ and $\mathrm{A}-\mathrm{US}$ associations prevented expression of the $\mathrm{X}-\mathrm{US}$ association. Therefore, in Phase 3 the $X-U S$ association, although latent in terms of direct 
response elicitation, was present. Consequently, one might expect that the X-US association would block expression of the $Y$-US association during testing. In fact, Williams found no evidence of blocking of $Y$ when $X$ had been subjected to blocking training, relative to a group in which $A$ had not been paired with the US in Phase 1 . That is, the blocked CS X did not prevent $Y$ from acquiring behavioral control. This finding is problematic for the comparator hypothesis if the comparator process is assumed to influence response generation rather than memory retrieval.

More recently, Rauhut, McPhee, and Ayres (1999) have reported similar findings which they also assert to be problematic for the comparator hypothesis' account of cue competition. In their studies, Rauhut et al. found that an overshadowed or blocked stimulus failed to block acquisition of behavioral control to a novel stimulus, a result replicating those of Williams (1996). Additionally, they found that a blocked or overshadowed stimulus failed to function as an effective second-order reinforcer when a previously neutral cue was followed by the target stimulus of a cue competition procedure. Again, these findings are problematic for the comparator hypothesis unless one assumes that the process that results in the attenuation of responding to a stimulus (i.e., the putative comparator process) also attenuates that stimulus' potential to modulate responding to other stimuli (e.g., Denniston et al., 2001). That is, a stimulus that fails to support robust behavioral control might also be unable to down-modulate (i.e., attenuate) responding to another stimulus, a possibility that we investigated in Experiments 3 and 4.

The present series of experiments is conceptually similar to those of Williams (1996) and Rauhut et al. (1999). In Experiment 1, we tested whether cue competition, overshadowing in this instance, is reversible without further target CS-US training. Overshadowing is a form of cue competition in which reduced behavioral control by a target CS $(X)$ is observed after training with CS $X$ in the presence of a second CS (A) which typically is more salient than $X(A X \rightarrow U S)$, relative to subjects for which $X$ alone was paired with the US (X $\rightarrow U S$; Pavlov, 1927). The attenuated responding observed as a result of overshadowing treatment has been explained as either an acquisition deficit (e.g., Mackintosh, 1975; Pearce, 1987; Pearce \& Hall, 1980; Rescorla \& Wagner, 1972) or a performance deficit ( Miller \& Matzel, 1988; also see Gallistel \& Gibbon, 2000). Toward preliminarily discriminating between these views, following overshadowing treatment in Experiment 1, we gave massive extinction of the overshadowing stimulus $(A \rightarrow$ noUS), which, according to the comparator hypothesis but not traditional acquisition-deficit models, should increase responding to the overshadowed stimulus. This result has been previously observed (e.g., Kaufman \& Bolles, 1981; Matzel, Schachtman, \& Miller, 1985) and was sought again here to establish its occurrence with the present preparation and parameters.

Experiment 2 investigated whether an overshadowed stimulus could serve as an effective secondary reinforcer for a previously neutral cue. This experiment was similar to that of Rauhut et al.'s Experiment 3 (1999). In their experiment, Rauhut et al. found that a blocked or overshadowed stimulus failed to support second-order responding. They interpreted this result as indicative of an acquisition deficit. To test this conclusion, Experiment 2 provided overshadowing training followed by second-order conditioning training in which the 
overshadowed stimulus served as the second-order reinforcer. We expected the overshadowed stimulus to fail to support second-order responding, as in Rauhut et al.'s Experiment 3, unless the overshadowing stimulus was previously extinguished.

Although the anticipated results of Experiments 1 and 2 are inconsistent with traditional models of associative acquisition (e.g., Rescorla \& Wagner, 1972), recent acquisition models designed to account for retrospective revaluation effects (e.g., recovery from blocking or overshadowing through posttraining extinction of the competing cue; Dickinson \& Burke, 1996; Van Hamme \& Wasserman, 1994) can readily accommodate our expected results. Hence, Experiments 3 and 4 were designed to differentiate the comparator hypothesis from these newer acquisition models. Experiment 3 served as a demonstration of recovery from overshadowing using a sensory preconditioning procedure, which was subsequently used in Experiment 4. (The rationale for using a sensory preconditioning procedure in Experiment 4 is discussed in Experiment 3.) In Experiment 4, we gave overshadowing training followed by blocking training in which the potential of the overshadowed stimulus to block responding to another novel stimulus was assessed. Based on Williams' (1996) results and those of Rauhut et al. (1999, Experiment 2), we anticipated little blocking by the overshadowed CS. However, following blocking training, some subjects received massive extinction of the overshadowing stimulus. The comparator hypothesis, framed in terms of modulation of retrieval rather than response generation, predicts that an overshadowed stimulus $(X)$ will fail to block responding to another stimulus $(Y)$ because the same process that renders CS X unable to support direct responding also attenuates its potential to down-modulate responding to CS Y (the target of blocking training). However, if the overshadowing stimulus (A) was extinguished following the blocking treatment, this should allow CS $X$ to evoke responding and to modulate responding to $Y$, resulting in less responding to $Y$ at test (relative to subjects who did not receive extinction of $A$ ).

\section{EXPERIMENT 1}

Experiment 1 was designed to further examine recovery from overshadowing. Although the procedures and hence the expected outcome were similar to that of prior reports (Kaufman \& Bolles, 1981; Matzel et al., 1985), Experiment 1 was necessary to demonstrate recovery from overshadowing with a conventional measure of overshadowing (i.e., excitatory responding to the overshadowed CS) using the exact parameters that we subsequently used in Experiment 2 , in which we used responding to a second-order stimulus as the dependent variable. The design of Experiment 1 is depicted in the top of Table 1. In Phase 1, four groups of rats were each exposed to two different sets of stimuli. Subjects in the overshadowing groups (OV-A, which received extinction of overshadowing stimulus $A$, and OV-B, which received extinction of overshadowing stimulus $B$ ) were presented with two separate stimulus compounds, $A X$ and $B Y$, which were each paired with a footshock US. CSs A and B were more salient than CSs $X$ and $Y$. As a result of this training, we anticipated that $A$ and $B$ would overshadow target $C S s ~ X$ and $Y$, respectively. Subjects in the control groups (Con-A and Con-B) received similar training, except that CS A was omitted. Thus, we expected overshadowing of $Y$, but not $X$. In Phase 2, subjects received massive extinction of either CS A (Groups OV-A and Con-A) or B (Groups 
OV-B and Con-B). The purpose of including training on BY and extinction of CS B was two-fold. Extinction of CS B equated Phase 2 experience between groups except for the specific association that was extinguished, and it examined the stimulus specificity of the recovery from overshadowing that we anticipated as a result of extinguishing an overshadowing stimulus (see also Miller, Barnet, \& Grahame, 1992). That is, according to the comparator hypothesis extinction of $B$ should have had no effect on conditioned responding to $X$ due to the absence of a B-X association. Based on the comparator hypothesis and previous empirical findings ( Kaufman \& Bolles, 1981; Matzel et al., 1985; Miller et al., 1992. R.R. Miller, R.C. Barnet and N.J. Grahame, Responding to a conditioned stimulus depends on the current associative status of other cues that were present during training of that specific stimulus. Journal of Experimental Psychology: Animal Behavior Processes 18 (1992), pp. 251-264. Miller et al., 1992), we expected overshadowing of CS X in Group OV-B, but not Group OV-A, and strong conditioned responding to $\mathrm{CS} X$ in both control groups.

Table 1. Design summary of Experiments 1 and 2

\begin{tabular}{|c|c|c|c|c|}
\hline Group & Phase 1 & Phase 2 & Test & \\
\hline \multicolumn{5}{|c|}{ Experiment 1} \\
\hline OV-A & $\mathrm{AX} \rightarrow \mathrm{US} / \mathrm{BY} \rightarrow \mathrm{US}$ & A- & $X$ ? & \\
\hline OV-B & $\mathrm{AX} \rightarrow \mathrm{US} / \mathrm{BY} \rightarrow \mathrm{US}$ & B- & $\mathrm{X}$ ? & \\
\hline Con-A & $\mathrm{X} \rightarrow \mathrm{US} / \mathrm{BY} \rightarrow \mathrm{US}$ & A- & $X ?$ & \\
\hline Con-B & $\mathrm{X} \rightarrow \mathrm{US} / \mathrm{BY} \rightarrow \mathrm{US}$ & B- & $\mathrm{X}$ ? & \\
\hline Group & Phase 1 & Phase 2 & Phase 3 & Test \\
\hline \multicolumn{5}{|c|}{ Experiment ? } \\
\hline OV-A & $\mathrm{AX} \rightarrow \mathrm{US} / \mathrm{BY} \rightarrow \mathrm{US}$ & A- & $\mathrm{Z} \rightarrow \mathrm{X}$ & $\mathrm{Z}$ ? \\
\hline OV-B & $\mathrm{AX} \rightarrow \mathrm{US} / \mathrm{BY} \rightarrow \mathrm{US}$ & B- & $\mathrm{Z} \rightarrow \mathrm{X}$ & $\mathrm{Z} ?$ \\
\hline Con-A & $\mathrm{X} \rightarrow \mathrm{US} / \mathrm{BY} \rightarrow \mathrm{US}$ & A- & $\mathrm{Z} \rightarrow \mathrm{X}$ & $\mathrm{Z}$ ? \\
\hline Con-B & $\mathrm{X} \rightarrow \mathrm{US} / \mathrm{BY} \rightarrow \mathrm{US}$ & B- & $\mathrm{Z} \rightarrow \mathrm{X}$ & $\mathrm{Z} ?$ \\
\hline
\end{tabular}

Note. A and B were either a flashing light or a high-frequency complex tone, counterbalanced; $X$ and $Y$ were either a white noise or a click train, counterbalanced; $Z$ was a low-frequency complex tone. CSs $A$ and $B$ were more salient than CSs $X$ and $Y$. - in Phase 2 represents nonreinforcement; $\rightarrow$ represents serial presentations with no gap between events. All CSs were $10 \mathrm{~s}$ in duration; the US was a 1-s footshock.

\section{Method}

\section{Subjects}

The subjects were 24 male and 24 female, experimentally naive, Sprague-Dawley derived rats from our SUNY-Binghamton breeding colony. Body weights ranged from 290-450 g for males and $190-300 \mathrm{~g}$ for females. The animals were individually housed in standard hanging, 
stainless-steel, wire-mesh cages in a vivarium maintained on a 16:8-h light:dark cycle. All training occurred approximately midway through the light portion of the cycle. Subjects were allowed free access to food in their home cages, whereas access to water was gradually decreased to $10 \mathrm{~min}$ per day prior to the initiation of the experiment. All subjects were handled for $30 \mathrm{~s}$ three times per week from weaning until the initiation of the study. Subjects were randomly assigned to one of four groups $(n s=12)$, counterbalanced for sex.

\section{Apparatus}

Two types of experimental chambers, designated $\mathrm{R}$ and $\mathrm{V}$, were used. Chamber $\mathrm{R}$ was rectangular in shape and measured $22.75 \times 8.25 \times 13.0 \mathrm{~cm}(\mathrm{l} \times \mathrm{w} \times \mathrm{h})$. The walls and ceiling of the chamber were constructed of clear Plexiglas, and the floors consisted of stainless-steel rods measuring $0.48 \mathrm{~cm}$ in diameter, spaced $1.5 \mathrm{~cm}$ center-to-center. The rods were connected by NE2-neon bulbs which allowed the delivery of constant-current footshock produced by a highvoltage $\mathrm{AC}$ circuit in series with a 1.0-M $\Omega$ resistor. Each of six copies of Chamber $\mathrm{R}$ was housed in a separate sound- and light-attenuating environmental enclosure. Chamber $\mathrm{R}$ was dimly illuminated by a 2.0-W (nominal at $120 \mathrm{VAC}$ ) house light driven at $60 \mathrm{VAC}$. The bulb was located on the inside wall of the environmental enclosure, approximately $30 \mathrm{~cm}$ from the center of the experimental chamber.

Chamber $V$ was a $25.5 \mathrm{~cm}$ long box in the shape of a vertical truncated-V. The chamber was $28 \mathrm{~cm}$ high, $21 \mathrm{~cm}$ wide at the top, and narrowed to $5.25 \mathrm{~cm}$ wide at the bottom. The ceiling was constructed of clear Plexiglas, the narrow end walls were black Plexiglas, and the side walls were stainless steel. The floor consisted of two $25.5 \mathrm{~cm}$ long parallel stainless steel plates, each $2 \mathrm{~cm}$ wide and separated by a 1.25-cm gap. A constant-current footshock could be delivered through the metal walls and floor of the chamber. Each of six copies of Chamber $V$ was housed in a separate sound- and light-attenuating enclosure. Chamber $V$ was illuminated by a 7-W (nominal at $120 \mathrm{VAC}$ ) bulb driven at $60 \mathrm{VAC}$. The bulb was mounted on the inside wall of the environmental enclosure, approximately $30 \mathrm{~cm}$ from the center of the experimental chamber, with the light entering the chamber primarily by reflection from the ceiling of the environmental enclosure. The light intensities inside Chambers $R$ and $V$ were approximately equal, despite the discrepancy in the light bulbs, because of differences between the chambers in the opaqueness of the walls.

Chambers $\mathrm{R}$ and $\mathrm{V}$ could each be equipped with a water-filled lick tube. When inserted, the lick tube extended $1 \mathrm{~cm}$ into a cylindrical drinking recess that was set into one of the Plexiglas end walls of the chamber. Each drinking recess was left-right centered with its bottom $1.75 \mathrm{~cm}$ above the floor of the chamber. The recess was $4.5 \mathrm{~cm}$ in diameter and $5 \mathrm{~cm}$ deep. An infrared photobeam was projected horizontally across the recess, $1 \mathrm{~cm}$ in front of the lick tube. To drink from the lick tube, a subject had to insert its head into the recess, thereby breaking the photobeam. By this means, we could monitor when subjects were accessing the lick tube. Three speakers, mounted on separate walls in each enclosure, could deliver the following auditory cues: a 6/s click train, a white noise, and a high-frequency complex tone (compound of 3000 and $3200 \mathrm{~Hz}$ ). The high-frequency tone was presented $10 \mathrm{~dB}$ above the ambient background of 
$74 \mathrm{~dB}(\mathrm{C})$, which was produced primarily by a ventilation fan on each environmental enclosure, whereas the white noise and click train were each presented at $6 \mathrm{~dB}(\mathrm{C})$ above background. Additionally, a flashing light stimulus ( $0.25 \mathrm{~s}$ on / $0.25 \mathrm{~s}$ off) could be presented. In Chamber R, the flashing light was produced by a 150-W bulb, whereas in Chamber $\mathrm{V}$ it was produced by a $60-\mathrm{W}$ bulb. The bulbs were located on the back wall of each environmental chest. Due to differences in the opaqueness of the chamber walls, these two stimuli produced approximately equal illumination in the $\mathrm{R}$ and $\mathrm{V}$ chambers. The high-frequency complex tone and the flashing light served as CSs A and B, counterbalanced within groups, and the click train and white noise served as CSs $\mathrm{X}$ and $\mathrm{Y}$, counterbalanced within groups. Except for testing, all CSs were $10 \mathrm{~s}$ in duration and the US was a 1-s, 0.7-mA footshock. Chamber type (R and V) was counterbalanced within groups.

\section{Procedure}

There were three critical phases in this experiment (see Table 1). In Phase 1, all subjects received overshadowing training with $B Y$ and either overshadowing or elemental training with $A X$ or $X$, respectively. In Phase 2, subjects in Groups OV-A and Con-A received massive extinction of CS A, whereas in Groups OV-B and Con-B subjects received massive extinction of CS B. Finally, in the test phase, conditioned responding to CS X was assessed by the potential of CS $X$ to suppress ongoing drinking.

Acclimation. Acclimation to the experimental chambers was conducted on Day 1 during a single 60-min session. Water-filled lick tubes were available and no nominal discrete stimuli were presented. This phase of the experiment served to establish a stable baseline level of drinking behavior, a departure from which would serve as the dependent variable during testing. Following acclimation, the water-filled lick tubes were removed from all chambers.

Overshadowing training (Phase 1). The first phase of training was conducted during four daily 60-min sessions on Days 2-5. All subjects received two daily AX-US pairings interspersed with two daily BY-US pairings, except for subjects in Groups Con-A and Con-B which received X-US rather than AX-US pairings. All CSs were $10 \mathrm{~s}$ in duration and were followed immediately at their termination by a US presentation. Two different stimulus presentation schedules were used. On the first schedule, trials occurred at 10, 20, 37, and 50 min into each daily session. On the second schedule, trials occurred at $9,28,37$, and 55 min into the session. These two schedules of stimulus presentation were alternated along with the patterns for trial type which were XYYX and YXXY.

Extinction (Phase 2). All subjects received 54 daily nonreinforced presentations of CS A (Groups OV-A and Con-A) or CS B (Groups OV-B and Con-B) on Days 6-9. The daily sessions were $135 \mathrm{~min}$ in duration and lick tubes were not available. Session duration was increased from 60 to $135 \mathrm{~min}$ in order to maintain adequate trial spacing between the CS presentations. The mean intertrial interval was $2.5 \mathrm{~min}$ (range $1-4 \mathrm{~min}$ ). 
Reacclimation. The lick tubes were returned to the chambers. All subjects were then reacclimated to the experimental context during two 60-min daily sessions (Days 10 and 11). These sessions served to stabilize baseline drinking following any potential disruption produced by the footshock USs.

Testing. Overshadowing was assessed during a single test session, using conditioned suppression of drinking as an assay of the behavioral control by CS X. During this test session, subjects were allowed to drink from the lick tubes for five cumulative seconds in the absence of any CS. Following this initial period of drinking, the test CS (X) was presented and the time to complete an additional five cumulative seconds of drinking was recorded. With this measure, long times to complete the second 5-cumulative seconds of drinking are indicative of conditioned fear to the CS. A 15-min ceiling was imposed on all suppression scores. Following the convention of our SUNY-Binghamton laboratory, any subject requiring greater than $60 \mathrm{~s}$ to complete the initial five cumulative seconds of drinking in the absence of the test CS, thus exhibiting a reluctance to drink in the test context, was scheduled to be eliminated from the data analysis. No subjects in this experiment met this criterion. Prior to statistical analysis, all suppression scores were converted to log (base 10) scores to better normalize the within-group distributions of scores, thereby allowing the use of parametric statistics. An alpha level of $p<.05$ was adopted for all statistical tests. Two subjects, one from Group OV-A and one from Group Con-A were eliminated from the study as a result of an experimenter error during testing.

\section{Results and discussion}

The principle findings from this study were that subjects in Group OV-B showed weak suppression to the overshadowed CS (X), indicative of overshadowing, whereas Group OV-A showed greater suppression to CS X, indicative of recovery from overshadowing (see left-hand side of Fig. 2). Furthermore, subjects in both control groups (Con-A and Con-B) showed strong conditioned responding to $\mathrm{CS} X$. These findings were confirmed with the following statistical analyses. 


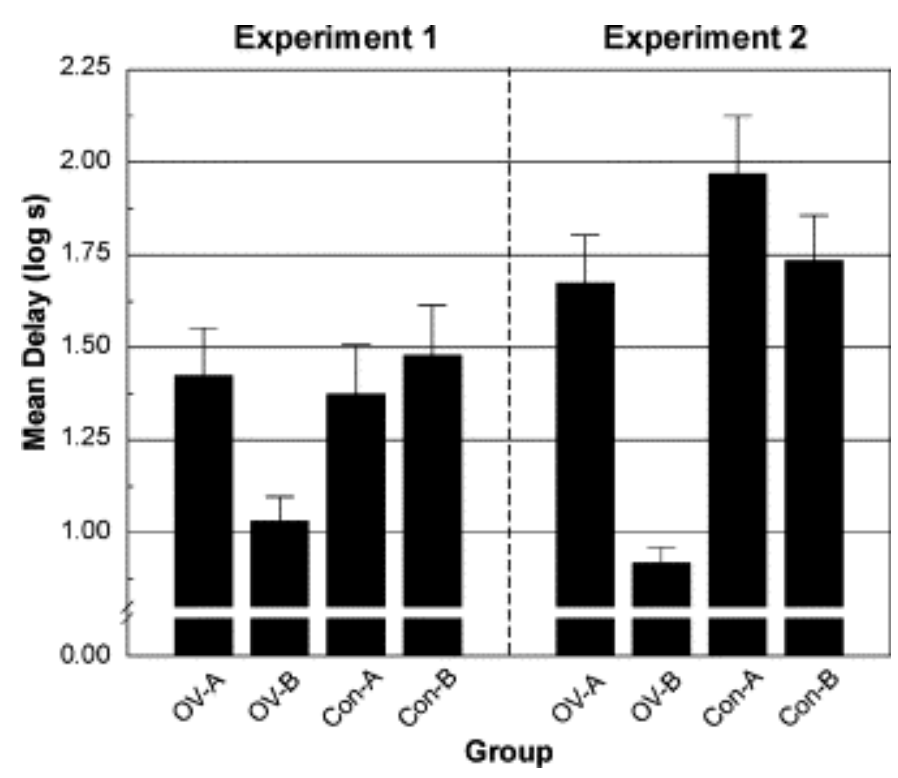

Fig. 2. Results of Experiments 1 and 2. Bars represent mean times (log $s$ ) to complete five cumulative seconds of drinking in the presence of target CS X (Experiment 1$)$ and second-order CS Z (Experiment 2). Higher scores are indicative of stronger stimulus control. Brackets represent standard errors of the means.

Analysis of the suppression scores in the presence of CS X were accomplished with a $2 \times 2$ Analysis of Variance (ANOVA) with Training Treatment (OV or Con) and Extinction (A or B) as factors.[1] This ANOVA revealed no main effect of Training Treatment, $F(1,42)=2.79, p>.10$, or Extinction, $F(1,42)=1.46, p>.23$, but did reveal an interaction, $F(1,42)=4.40, p<.05$. Planned comparisons using the overall error term from the latter $2 \times 2$ ANOVA were conducted to investigate the source of the interaction. Comparison of suppression scores from Groups OV-B and Con-B revealed a significant difference between groups, $F(1,42)=7.41, p<.01$, indicative of overshadowing of CS X in Group OV-B relative to Group Con-B. Additionally, Group OV-A suppressed more than did Group OV-B, $F(1,42)=5.47, p<.05$, a finding indicative of stimulusspecific recovery from overshadowing.

The results of Experiment 1 demonstrate that overshadowing can be reversed without further training with the target $\mathrm{CS}(\mathrm{X})$. Support for this assertion can be found in the high level of conditioned suppression in Group OV-A, which received extinction of the overshadowing stimulus in Phase 2. Posttraining massive extinction of CS A enhanced suppression by this group despite the absence of further X-US pairing. Moreover, the stimulus specificity of this effect (compare with Group OV-B) affirms the associative basis of this recovery from overshadowing. These results are problematic for traditional acquisition-deficit models of associative learning (e.g., Rescorla \& Wagner, 1972), which posit that overshadowing results from a failure to acquire a strong X-US association. In contrast, these results are readily accounted for by the comparator hypothesis, which claims that the response deficit observed following overshadowing training stems from strong target CS-comparator stimulus $(\mathrm{X}-\mathrm{A})$ and comparator stimulus-US (A-US) associations. Weakening either of these links through 
extinction of the comparator stimulus presumably decreases the strength of the indirectly activated US representation, thereby enhancing excitatory behavioral control by CS X. The results are also compatible with recent acquisition-focused models designed to account for retrospective revaluation (discussed later).

Notably, these results stand in contrast to those of Rauhut et al. (1999, Experiments 4 \& 5), who failed to observe an attenuation of the overshadowing effect through posttraining extinction of the overshadowing stimulus. Their failure to observe the effect found in our Experiment 1 might question the generality of recovery from cue competition following extinction of the competing cue; however, numerous other researchers have observed associative deflation effects using a variety of procedures, tasks, and species. For example, Dickinson and Charnock (1985) reported alleviation of cue competition through posttraining extinction of the overshadowing stimulus in instrumental responding, Kaufman and Bolles (1981) and Matzel et al. (1985) in Pavlovian overshadowing, Blaisdell, Gunther, and Miller (1999) in Pavlovian blocking, Shanks (1985) and Wasserman and Berglan (1998) in blocking of causal judgment, Cole, Barnet, and Miller (1995) in the relative stimulus validity effect, Blaisdell, Denniston, and Miller (2001) in the overexpectation effect, Best, Dunn, Batson, Meachum, and Nash (1985), Hallam, Matzel, Sloat, and Miller (1990), and Lysle and Fowler (1985) in Pavlovian conditioned inhibition, Miller, Hallam, Hong, and Dufore (1991) in differential conditioned inhibition, and Kasprow, Schachtman, and Miller (1987), and Schachtman, Brown, Gordon, Catterson, and Miller (1987) in negative contingency conditioned inhibition.

One potential explanation for Rauhut et al.'s (1999) failure to observe recovery from overshadowing and blocking in their Experiments 4 and 5 stems from a procedural difference between their experiments and the current Experiment 1. In their experiments, CS A, the overshadowing stimulus was the termination of white noise and a ventilation fan that were normally on. It is possible that effectiveness of their extinction treatment, and hence the likelihood of observing recovery from cue competition, was undermined by their choice of stimuli. That is, the salience of a stimulus-absent cue might decrease following repeated exposure (32 2-min presentations in their Experiment 4 and 102 2-min presentations in their Experiment 5) more rapidly than the salience of a stimulus-present cue, thereby leading to less effective extinction and consequently weaker recovery from overshadowing. In contrast, in the present Experiment 1 we used a bright flashing light as CS A which was presented for only $10 \mathrm{~s}$ per exposure. If the brief assertion of a stimulus is more salient than the prolonged (2 $\mathrm{min}$ ) omission of a stimulus, this likely provides an explanation for their failure to observe a recovery from overshadowing effect. This possibility is supported by Rauhut et al.'s observation of spontaneous recovery of responding to CS A in their experiments (see Rauhut et al., 2000, for a discussion of this possibility).

Additional challenges to the performance-deficit account of cue competition are provided by a recent series of experiments by Holland (1999). Holland found that posttraining extinction of the competing cue failed to attenuate blocking and overshadowing. However, all of his experiments failed to control for configuring and generalization decrement. That is, the weak responding to CS $X$ following $A X-U S$ overshadowing treatment might reflect generalization decrement from $A X$ to $X$ rather than associative cue competition. This possibility is supported by the effect of his 
extinction treatment. In each of his seven experiments, posttraining extinction treatment of CS A attenuated responding to CS X. This observation is consistent with a generalization decrement account of his results, in that extinction of CS A would be expected to result in less generalized excitation from the AX compound to CS X. The present series of experiments similarly failed to control for generalization decrement; however, we obtained the expected recovery effect (i.e., extinction of CS A enhanced responding to CS X), which would not be anticipated by a configuring and generalization decrement account of the present results.

Failures to observe recovery from cue competition following extinction of the competing cue are not limited to the studies of Holland (1999) and Rauhut and Rauhut, For example, in several experiments Schachtman, Kasprow, Meyer, Bourne, and Hart (1992) failed to observe recovery from overshadowing following posttraining extinction of the overshadowing stimulus in a conditioned taste aversion preparation. Similarly, Speers, Gillan, and Rescorla (1980) found that extinction of the blocking stimulus attenuates rather than enhances responding to the blocked stimulus in a conditioned taste aversion preparation (presumably due to a strong withincompound association between the blocking and blocked stimuli). Thus, the results of Rauhut and Rauhut and of Holland (1999) are not anomalous. Although many of the failures to observe recovery from cue competition have used a conditioned taste aversion preparation (e.g., Schachtman et al.; Speers et al.), it seems implausible that it is the preparation per se that is responsible for determining whether recovery or mediated extinction will be observed. Instead, it is probably some procedural detail of the training that is responsible for these discrepant outcomes. For example, Rauhut et al. (2000) attempted to maximize the likelihood of observing recovery from blocking by providing posttraining conditioned inhibition treatment following blocking training. This training was intended to reduce responding to the blocking stimulus by establishing it as a conditioned inhibitor, thereby weakening the A-US association. Despite conditioned inhibition treatment, Rauhut et al. failed to observe recovery from blocking. As discussed by Rauhut et al., one of the many procedural differences between their procedure and ours was the duration of the CS. Their studies used a 2-min CS which consisted of the omission of an otherwise present stimulus, whereas the present study used a 10-s CS which consisted of the presentation of an otherwise absent stimulus. Regardless of the actual source of this discrepancy, the present Experiment 1 provides a demonstration that recovery from overshadowing can be obtained with the current procedures and parameters.

\section{EXPERIMENT 2}

Experiment 1 demonstrated that overshadowing is sometimes reversible without further training with the target CS. Posttraining extinction (associative deflation) of the competing cue, such as was done in Experiment 1, is one method of contrasting acquisition and expression deficit accounts of cue competition; however, other assays exist, for example second-order conditioning (Pavlov, 1927). In their Experiment 3, Rauhut et al. (1999) examined the potential of a blocked or overshadowed stimulus to support second-order conditioning and found that a stimulus subjected to cue competition failed to serve as an effective second-order reinforcer, 
whereas a control cue that had not been subjected to cue competition did. Rauhut et al. interpreted these results as problematic for a performance deficit account of cue competition.

Although the comparator hypothesis is notably unable to account for the acquisition of secondorder responding, in our experience the comparator process often requires some minimal number of training trials before it is engaged, and prior to this second-order responding is observed. Although second-order conditioning is beyond the domain of the comparator hypothesis, we here assume, as do all other models, that the strength of responding to a second-order CS should depend upon the magnitude of responding supported by the first-order CS. That is, if an overshadowed cue fails to elicit robust conditioned responding, then that cue should fail to serve as an effective reinforcer in second-order conditioning, provided that the process that attenuates retrieval of the overshadowed stimulus-US association during direct testing with the overshadowed cue is also active during second-order conditioning treatment. Furthermore, if overshadowing results from an expression deficit, then massive extinction of the overshadowing stimulus ( $A$ ) should not only enhance responding to the overshadowed stimulus $X$ (as in Experiment 1), but should also enhance the potential of CS X to serve as a secondary reinforcer. Experiment 2 tested this prediction using the same procedure as in Experiment 1 except that all subjects received an additional phase of training in which second-order conditioning was given in Phase 3 (i.e., $Z \rightarrow X$ ). Specifically, we tested whether an overshadowed CS $(X)$ could support second-order conditioning following extinction of the overshadowing stimulus (A), or following extinction of another (irrelevant) overshadowing stimulus (B). We expected that the continued presence of a strong A-US association following extinction of CS B (Group OV-B) would attenuate both conditioned responding to CS X (as demonstrated in Experiment 1 ) and the potential of CS X to support second-order conditioning. More importantly, we expected that providing massive extinction of CS A (Group OV-A) would reduce overshadowing (as demonstrated in Experiment 1 ) and consequently enhance the potential of CS X to support second-order conditioning in Group OV-A. Thus, we expected to observe strong second-order responding to CS Z in all groups except Group OV-B.

\section{Method}

\section{Subjects and apparatus}

The subjects were 48 male (234-450 g) and 48 female (200-305 g), 80-120-day-old, naïve Sprague-Dawley descended rats from our SUNY-Binghamton breeding colony. Subjects were maintained and housed as in Experiment 1. Subjects were randomly assigned to one of four groups (ns=24), counterbalanced for sex. The apparatus was the same as in Experiment 1, except for the addition of a low-frequency complex tone (300 and $320 \mathrm{~Hz}$ ) which could be presented at $8 \mathrm{~dB}(\mathrm{C})$ above background, and which served as CS Z. 


\section{Procedure}

The procedure of Experiment 2 was identical to that of Experiment 1, except for the addition of a third phase of training prior to testing. Phase 3 (Day 10) consisted of four nonreinforced $Z \rightarrow X$ pairings, in which termination of $Z$ coincided with onset of $X$. These presentations occurred 10 , 20,37 , and 50 min into the 60-min session. Lick tubes were not available during this phase of the study. Reacclimation (Day 11) and Testing (Day 12) were the same as in Experiment 1, except that subjects were tested for conditioned suppression to CS Z, rather that to CS X. That is, the time to complete 5-cumulative seconds of drinking in the presence of $Z$ served as the dependent variable in this study. As in Experiment 1, no subject required more than $60 \mathrm{~s}$ to complete five cumulative seconds of drinking prior to the onset of the test stimulus. One subject from Group OV-B was eliminated from the data analysis as a result of an equipment failure during testing. Data transformation and statistical analysis were the same as in Experiment 1.

\section{Results and discussion}

The primary result from Experiment 2 was that the overshadowed stimulus failed to support second-order conditioning (Group OV-B) unless the overshadowing stimulus (A) had been extinguished prior to second-order conditioning (Group OV-A). The mean log times to complete 5 -cumulative seconds of drinking in the presence of CS Z are depicted in Fig. 2. As can be seen in the figure, Group OV-B showed weak behavioral control by Z, whereas Group OV-A, which received extinction of $C S A$, showed strong conditioned responding to $Z$ that was nearly equivalent to that observed in the control groups. These findings were confirmed with the following statistical analyses.

Analysis of the (log) times to complete 5-cumulative seconds of drinking in the presence of the test CS (Z) was accomplished with a 2×2 ANOVA with Training Treatment (OV or Con) and Extinction ( $A$ or $B$ ) as factors.1 This ANOVA revealed a main effect of Training Treatment, $F(1,91)=20.65, p<.001$, and of Extinction, $F(1,91)=16.42, p<.001$, as well as an interaction of these factors, $F(1,91)=4.55, p<.05$. Planned comparisons using the error term from the latter $2 \times 2$ ANOVA were then conducted to identify the source of this interaction. These comparisons revealed that Group OV-B suppressed less to $Z$ than did Group Con-B, $F(1,91)=22.06, p<.001$, a finding indicative of the overshadowed stimulus failing to support second-order conditioning in Group OV-B following extinction of an overshadowing stimulus different than that with which CS $X$ was trained. Importantly, Group OV-A suppressed more to CS Z than did Group OV-B, $F(1,91)=18.93, p<.001$, indicating that massive extinction of CS A in Group OV-A allowed the overshadowed stimulus to support second-order conditioning. Additionally, there was no difference in conditioned suppression to CS Z between groups OV-A and Con-A, $F(1,91)=2.94$, $\mathrm{p}>.05$, indicating that the recovery effect in Group OV-A was nearly complete. Finally, there was no difference in suppression between Groups Con-A and Con-B, $F(1,91)=1.86, p>.15$, demonstrating that extinction of CS B did not generalize to CS X.

One unanticipated finding in Experiment 2 was the increased conditioned suppression to CS Z in Groups OV-A, Con-A and Con-B relative to the same groups in Experiment 1 . Whether this 
difference reflects abnormally high conditioned suppression in Experiment 2 or attenuated suppression in Experiment 1 remains unclear, as does the source of this between-experiment difference. Importantly, the greater overall responding in Experiment 2, relative to Experiment 1, does not appear to undermine the conclusions of either study.

In summary, Experiment 2 demonstrated that an overshadowed stimulus could support secondorder conditioning only when the overshadowing stimulus had first been massively extinguished. The absence of robust second-order responding in Group OV-B is consistent with Rauhut et al.'s (1999) findings that an overshadowed or blocked CS failed to support second-order conditioning. However, we additionally determined that posttraining extinction of the overshadowing CS allowed the overshadowed CS to support second-order conditioning. This last observation calls into question Rauhut et al.'s interpretation of their results, namely that cue competition results from an associative acquisition deficit.

Notably, the results of Experiments 1 and 2 are readily explicable by more recent associative acquisition models designed to explicitly accommodate retrospective revaluation effects (e.g., Dickinson \& Burke, 1996; Van Hamme \& Wasserman, 1994). Van Hamme and Wasserman's model, a modification of the Rescorla-Wagner model ( Rescorla \& Wagner, 1972), changed the manner in which non-presented cues are treated by the Rescorla-Wagner growth equation. According to the original Rescorla-Wagner model, only cues present on a given trial are eligible for increments or decrements in associative strength as a result of that trial (i.e., associability [a] for a non-presented CS was assumed to equal zero, thereby preventing any change in associative strength). However, Van Hamme and Wasserman proposed that $\alpha$ for an absent cue has a negative value (on trials during which a stimulus with a within-compound association to the omitted CS is presented). The consequence of this modification is that a cue can undergo increases or decreases in associative strength, even when that cue is absent on the trial in question. That is, further training or extinction of a competing cue can result in associative 'retrospective revaluation' of the absent target cue.

Similarly, Dickinson and Burke (1996) have extended Wagner's (1981) SOP model allowing it to account for retrospective revaluation effects. According to Wagner's original model, when a stimulus is presented, part or all of the event representation is activated from I (the inactive memory state) into A1 (the high activation memory state). However, when an associate of a stimulus is presented, the stimulus representation is activated into State A2 (the low activation memory state). According to Wagner's original SOP model, associative acquisition occurs when event representations coincide in the A1 state. In contrast, no learning occurs when two stimulus representations simultaneously occupy the A2 state.

Dickinson and Burke's (1996) modification of Wagner's (1981) SOP model allows for the strengthening of an excitatory association between two stimuli when their representations are both simultaneously activated in the A2 state as well as simultaneously in the A1 state. Applied to recovery from overshadowing, extinction of the overshadowing CS (A) following overshadowing training results in CS A being represented in the A1 state (when it is presented), which activates representations of both $\mathrm{CS} X$ and the US into the A2 state (as a consequence of strong $A-X$ and A-US associations). Simultaneous activation of CS X and the US in the A2 
state should lead to a strengthening of the X-US association, thereby attenuating the response deficit normally observed following overshadowing treatment (as demonstrated in Experiment 1). Applied to Experiment 2, extinction of the overshadowing stimulus should enhance the associative strength of the overshadowed stimulus, thereby allowing it to support second-order conditioning. Thus, Dickinson and Burke's model can readily account for the results of Experiments 1 and 2. Experiments 3 and 4 were designed to contrast the predictions of acquisition-focused models of retrospective revaluation effects (i.e., Dickinson \& Burke; Van Hamme \& Wasserman, 1994) with performance-deficit accounts of cue competition effects (e.g., Miller \& Matzel, 1988; Miller \& Schachtman, 1985).

\section{EXPERIMENT 3}

In Experiment 3, subjects received overshadowing training followed by extinction of the overshadowing stimulus, all embedded within a sensory preconditioning procedure (Brogden, 1939). This experiment was designed to demonstrate stimulus-specific recovery from overshadowing in a sensory preconditioning procedure, so that the preparation could subsequently be used in Experiment 4. As previously discussed, Williams (1996) found that a blocked stimulus was unable to block acquisition of responding to a previously neutral cue. That is, following blocking treatment $(A \rightarrow U S$ prior to $A X \rightarrow U S)$, CS $X$ failed to block acquisition of behavioral control by $C S Y$ when animals received $X Y \rightarrow U S$ training. Similarly, Rauhut et al. (1999, Experiment 2) found that, following overshadowing or blocking treatment overshadowed or blocked stimulus $X$ failed to block acquisition of behavioral control by previously neutral stimulus $Y$ (i.e., $X Y \rightarrow U S$ training). These authors attributed their results to an associative acquisition deficit. To further test this interpretation, Rauhut et al. examined whether extinction of CS X's blocking stimulus (Experiment 4) or overshadowing stimulus (Experiment 5) prior to blocking treatment of $Y(X Y \rightarrow U S)$ would allow $C S X$ to block acquisition of behavioral control by CS Y. Their results found no effect of extinguishing the competing cue. That is, extinction of the CS A failed to allow CS X to block acquisition of behavioral control by CS Y. Importantly, Rauhut et al. also failed to observe an attenuation of the blocking and overshadowing effects through extinction of the competing cue (Experiments 4 and 5, respectively).

The results of Williams (1996) and Rauhut et al. (1999) as well as those of the present Experiments 1 and 2 are inconsistent with the comparator hypothesis if the comparator process is framed purely in terms of modulating response generation. However, they can be accounted for within the framework of the comparator hypothesis if the comparison down-modulates retrieval of the target CS-US association for purposes of using the association for either response generation or as a comparator association for other target CSs. One way to conceptualize the comparator process as influencing retrieval of memories is similar to that envisioned by Wagner's (1981) SOP model in which stimulus elements reside in active (A1) and inactive (A2) states. That is, these inactive associations might exert an influence on other associations at a stage of processing that is pre-retrieval. With such an emphasis on retrieval, the performance-focused comparator hypothesis (as well as the new acquisition-focused models capable of accounting for retrospective revaluation) would not anticipate that a blocked 
or overshadowed stimulus could subsequently block acquisition of behavioral control by a neutral stimulus. The acquisition-focused models of retrospective revaluation posit that responding is monotonically related to associative value. Hence, if a stimulus supports weak conditioned responding, it presumably has a weak association with the US and consequently should be unable to block acquisition of association to the US by another stimulus. Similarly, the comparator hypothesis posits that if a stimulus fails to support robust responding as a consequence of a strong target stimulus-comparator stimulus and comparator stimulus-US associations, then it should be unable to down-modulate responding to another stimulus. That is, the potential of a stimulus to serve as an effective comparator stimulus depends upon the response potential of that stimulus. To summarize, Rauhut et al.'s finding that an overshadowed or blocked stimulus failed to block acquisition of behavioral control by a neutral stimulus is not surprising given the absence of a recovery effect in their preparation. Had Rauhut et al. observed recovery from initial overshadowing and blocking, then both the acquisition-focused retrospective revaluation models and the comparator hypothesis would anticipate subsequent blocking by the initially overshadowed or blocked cue.

Experiments 3 and 4 used an experimental design similar to that of Rauhut et al. (1999), except that these experiments were designed to differentiate between the acquisition-focused models of retrospective revaluation and the comparator hypothesis. One difference between the acquisition-focused models of Dickinson and Burke (1996) and Van Hamme and Wasserman (1994) and the performance-focused model of Miller and Matzel (1988) is the predicted effect of extinguishing a competing cue either before or after blocking treatment that involves a competing (companion) cue which itself has previously been blocked or overshadowed. Following overshadowing training (i.e., $A X \rightarrow U S$ ), if the overshadowing stimulus were extinguished (i.e., $A \rightarrow$ noUS) prior to blocking treatment of CS Y (i.e., $X Y \rightarrow U S$ ), thereby allowing CS X to support robust conditioned responding, both families of models would anticipate a strong blocking effect in which CS Y would fail to support robust conditioned responding. However, the acquisition-focused models of retrospective revaluation do not predict retrospective blocking of CS Y when extinction of CS X's competing cue (A) occurs following $\mathrm{XY} \rightarrow \mathrm{US}$ blocking treatment. That is, the acquisition-focused models of retrospective revaluation predict that following $A X \rightarrow U S$ overshadowing treatment of CS $X$ and $X Y \rightarrow U S$ blocking training of CS Y, extinction of CS A either should have no effect on responding to CS Y (due to the lack of an A-Y within-compound association), or should result in an increase in conditioned responding to CS $Y$ (that, if one assumes during extinction of CS A, CS A activates a representation of $C S X$ which in turn activates a representation of CS $Y$ ). In contrast, if the comparator process is assumed to influence the retrieval of event representations rather than response generation, then following $X Y \rightarrow U S$ blocking treatment of $C S Y$, the strong A-US association should attenuate retrieval of the $X-U S$ association, thereby attenuating the potential of CS X to down-modulate responding to CS Y. Provided that extinction of CS A after XY $\rightarrow$ US blocking treatment enhances retrieval of the $X-U S$ association, such extinction treatment should also cause the $X-U S$ representation to down-modulate responding to CS Y. Hence, the comparator hypothesis differentiates itself from the acquisition-focused models of retrospective revaluation in predicting retrospective blocking of CS Y when extinction of the competing cue (A) is provided following blocking training. Notably, the comparator account assumes that CS A 
retains its status as the primary comparator stimulus for CS $X$ despite the absence of $A$ on the $X Y \rightarrow U S$ trials. However, with the number of $X Y \rightarrow U S$ trials not exceeding the number of $A X \rightarrow U S$ trials and $A$ being more salient than $Y$, this assumption appears plausible.

The designs of Experiments 3 and 4 are depicted in Table 2. The purpose of Experiment 3 was to demonstrate stimulus-specific recovery from overshadowing in the precise sensory preconditioning procedure that was used in Experiment 4. Experiment 4 was designed to assess whether an overshadowed stimulus $(X)$ could retrospectively block responding to a previously neutral cue $(\mathrm{Y})$ when extinction of the competing cue was provided following $\mathrm{XY} \rightarrow \mathrm{US}$ blocking treatment. These experiments used a sensory preconditioning procedure because prior studies (e.g., Denniston, Miller, \& Matute, 1996; Miller \& Matute, 1997) have found that posttraining inflation procedures are less effective in first-order conditioning than in sensory preconditioning (a finding inexplicable in terms of any contemporary model). Specifically, Experiment 4 examined the effects of posttraining deflation of CS A, which was expected to increase the response potential of CS X (assessed by Experiment 3), which in turn should attenuate responding to CS Y (assessed by Experiment 4). This latter effect is an associative inflation effect, which tends to be more difficult to obtain in first-order conditioning. The studies of Denniston et al. and Miller and Matute found that, once a stimulus controls responding, it is relatively immune to cue competition effects. For example, Miller and Matute found that following AX-US pairings, further A-US training failed to attenuate responding to CS $X$ (i.e., failed to produce backward blocking), but that if the same procedure were imbedded within a sensory preconditioning procedure, robust backward blocking was observed. Thus, we anticipated that if Experiment 4 were performed in first-order conditioning, the acquired biological significance of CS Y would work against the likelihood of observing retrospective revaluation of CS Y through extinction of the overshadowing stimulus $(A)$; sensory preconditioning circumvents this problem.

Table 2. Design summary of Experiments 3 and 4

\begin{tabular}{|c|c|c|c|c|c|}
\hline Group & Phase 1 & Phase 2 & Phase 3 & Test & \\
\hline \multicolumn{6}{|c|}{ Experiment 3} \\
\hline OV-A & $\mathrm{AX} \rightarrow \mathrm{S} / \mathrm{BY} \rightarrow \mathrm{S}$ & A- & $\mathrm{S} \rightarrow \mathrm{US}$ & $X$ ? & \\
\hline OV-B & $\mathrm{AX} \rightarrow \mathrm{S} / \mathrm{BY} \rightarrow \mathrm{S}$ & B- & $\mathrm{S} \rightarrow \mathrm{US}$ & $X$ ? & \\
\hline Con-A & $\mathrm{X} \rightarrow \mathrm{S} / \mathrm{BY} \rightarrow \mathrm{S}$ & A- & $\mathrm{S} \rightarrow \mathrm{US}$ & $X$ ? & \\
\hline Con-B & $\mathrm{X} \rightarrow \mathrm{S} / \mathrm{BY} \rightarrow \mathrm{S}$ & B- & $\mathrm{S} \rightarrow \mathrm{US}$ & $X ?$ & \\
\hline Group & Phase 1 & Phase 2 & Phase 3 & Phase 4 & Test \\
\hline \multicolumn{6}{|c|}{ Experiment 4} \\
\hline $\mathrm{OV}-\mathrm{A}$ & $\mathrm{AX} \rightarrow \mathrm{S}$ & $\mathrm{XY} \rightarrow \mathrm{S}$ & A- & $\mathrm{S} \rightarrow \mathrm{US}$ & $Y ?$ \\
\hline OV-Cxt & $A X \rightarrow S$ & $\mathrm{XY} \rightarrow \mathrm{S}$ & Context & $\mathrm{S} \rightarrow \mathrm{US}$ & $Y ?$ \\
\hline Con-A & $X \rightarrow S$ & $\mathrm{XY} \rightarrow \mathrm{S}$ & A- & $\mathrm{S} \rightarrow \mathrm{US}$ & Y? \\
\hline Con-Cxt & $X \rightarrow S$ & $\mathrm{XY} \rightarrow \mathrm{S}$ & Context & $\mathrm{S} \rightarrow \mathrm{US}$ & Y? \\
\hline
\end{tabular}

Note. In Experiment 3, A and B were either a flashing light or a high-frequency complex tone, 
counterbalanced; $X$ and $Y$ were either a white noise or a click train, counterbalanced. CSs $A$ and $B$ were more salient than CSs $X$ and $Y$. - in Phase 2 of Experiment 3 and Phase 3 of Experiment 4 represents nonreinforcement; $\rightarrow$ represents serial presentations with no gap between events. In Experiment 4, A was a high-frequency complex tone; $X$ was a white noise; $Y$ was a click train; $S$ (the surrogate for the US) was a buzz stimulus. In Experiment 4, CS A was more salient than CS $X$; CSs $X$ and $Y$ were of equal salience. In both experiments, CSs $A, B, X$, and $Y$ were $10 \mathrm{~s}$ in duration; $\mathrm{S}$ was $5 \mathrm{~s}$ in duration; the US was a 1-s footshock.

The design of Experiment 3 was the same as that of Experiment 1, except that all training was embedded in a sensory preconditioning procedure. In Phase 1, the overshadowing groups (OV$A$ and $O V-B$ ) received overshadowing training in which subjects were presented with two stimulus compounds, $A X$ and $B Y$, which were each followed by the surrogate for the US, Stimulus $S$, which was later paired with footshock. Subjects in the control groups (Con-A and Con-B) received similar training except that CS A was omitted. In Phase 2, subjects in Groups OV-A and Con-A received massive extinction of CS A, whereas subjects in Groups OV-B and Con-B received massive extinction of CS B. Prior to testing, we paired CS S with the footshock US. As in Experiment 1, we expected to observe strong conditioned responding to $C S X$ in Group OV-A (relative to Con-A), indicative of recovery from overshadowing, but not in Group OV-B (relative to Con-B), thereby demonstrating stimulus specificity of recovery from overshadowing within a sensory preconditioning procedure.

\section{Method}

\section{Subjects and apparatus}

The subjects were 24 male (322-460 g) and 24 female (208-295 g), 80-120-day-old, naïve, Sprague-Dawley descended rats from our SUNY-Binghamton breeding colony. Subjects were maintained and housed as in Experiment 1 . They were randomly assigned to one of four groups (ns=12), counterbalanced for sex. The apparatus was the same as in Experiment 1, except for the addition of a buzzer stimulus which could be presented at $8 \mathrm{~dB}(\mathrm{C})$ above background, which served as the surrogate (S) for the US.

\section{Procedure}

The procedure of Experiment 3 was similar to that of Experiment 1, except that all phases of training were embedded within a sensory preconditioning procedure.

Acclimation. Acclimation to the apparatus was conducted the same as in Experiment 1.

Overshadowing training (Phase 1). The first phase training was conducted during four daily 60min sessions on Days 2-5. All subjects received two daily $A X \rightarrow S$ pairings interspersed with two daily $B Y \rightarrow S$ pairings, except for subjects in Groups Con-A and Con-B which received $X \rightarrow S$ rather than $A X \rightarrow S$ pairings. All CSs were $10 \mathrm{~s}$ in duration and were followed immediately at 
their termination by presentation of $\mathrm{S}$ for $5 \mathrm{~s}$. Two different stimulus presentation schedules were used as in Experiment 1.

Extinction (Phase 2). All subjects received 54 daily nonreinforced presentations of CS A (Groups OV-A and Con-A) or CS B (Groups OV-B and Con-B) on Days 6-9. This phase of training was the same as in Experiment 1.

S-US Training (Phase 3). All subjects received four $\mathrm{S} \rightarrow \mathrm{US}$ pairings during a single 60-min session on Day 10. US onset occurred at Stimulus $S$ termination. The US was a 1-s, 0.9-mA footshock. The increase in footshock intensity over that of Experiment 1 was intended to partially compensate for sensory preconditioning yielding weaker conditioned responding than first-order conditioning. Trials occurred at 10, 20, 37, and 50 min into the session. Lick tubes were not available during these sessions.

Reacclimation and testing. Reacclimation (Day 11) and Testing (Day 12) were the same as in Experiment 1. Two animals from Group OV-A were excluded from the data analysis for failing to complete the five cumulative seconds of drinking prior to test stimulus onset within $60 \mathrm{~s}$. Data transformation and statistical analysis were the same as in Experiments 1 and 2.

\section{Results and discussion}

The results of Experiment 3 replicated the basic overshadowing effect and more importantly demonstrated recovery from overshadowing through posttraining extinction of the overshadowing cue within a sensory preconditioning procedure (see Fig. 3). Specifically, subjects in Group OV-B showed weak conditioned responding to CS X at test (relative to Group Con-B), demonstrating the basic overshadowing effect, whereas subjects in Group OV-A showed strong conditioned responding to CS X (relative to Group OV-B) demonstrating recovery from overshadowing. Additionally, the lack of recovery observed in Group OV-B demonstrates stimulus specificity of recovery from overshadowing (i.e., extinction of overshadowing stimulus $B$ had little or no effect on responding to CS $X$ which was overshadowed by CS A). These results were confirmed with statistical analyses. 


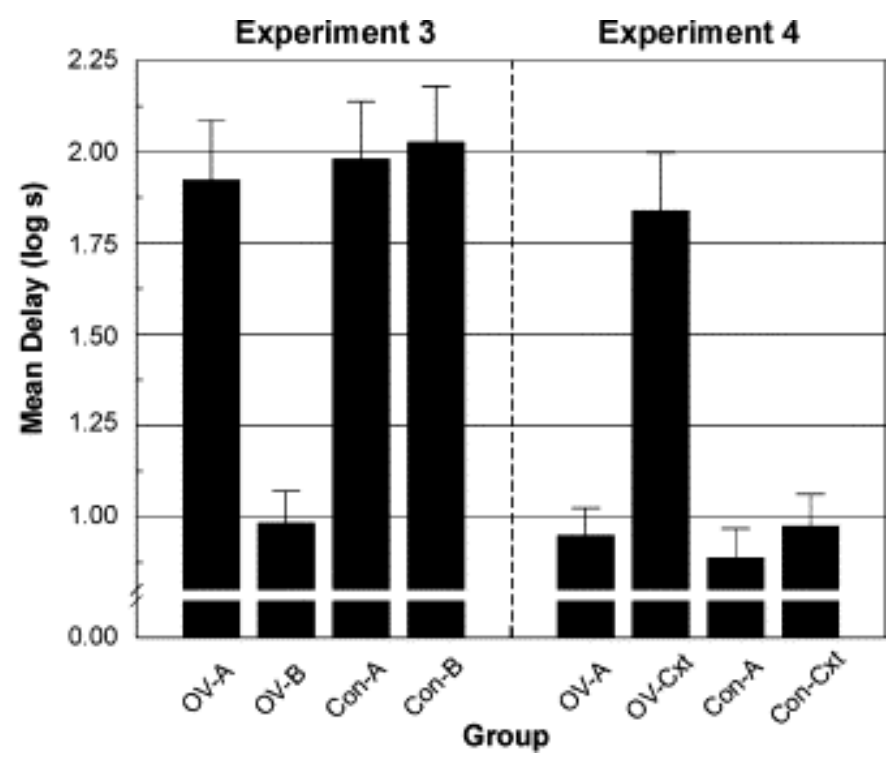

Fig. 3. Results of Experiments 3 and 4. Bars represent mean times (log s) to complete five cumulative seconds of drinking in the presence of target CS X (Experiment 3) and target CS Y (Experiment 4). Higher scores are indicative of stronger stimulus control. Brackets represent standard errors of the means.

A 2×2 ANOVA with Training Treatment (OV or Con) and Extinction ( $\mathrm{A}$ or $\mathrm{B}$ ) as factors was conducted on the $(\mathrm{log})$ times to drink in the presence of CS $X .^{-1}$ This ANOVA revealed a main effect of Training Treatment, $F(1,42)=14.63, p<.001$, and of Extinction, $F(1,42)=9.60, p<.01$, as well as an interaction, $F(1,42)=11.72, p<.01$. Planned comparisons using the error term from the latter ANOVA were then conducted to identify the source of this interaction. These comparisons revealed that Group OV-B suppressed less than did Group Con-B $F(1,42)=27.58, p<.001$, demonstrating the basic overshadowing effect in a sensory preconditioning procedure. Additionally, Group OV-A suppressed more than did Group OV-B, $F(1,42)=20.48, p<.001$, indicative of recovery from overshadowing. Thus, the results of Experiment 3 replicate those of Experiment 1, but now within a sensory preconditioning procedure.

\section{EXPERIMENT 4}

Experiment 4 was designed to differentiate between the performance-focused account of retrospective revaluation on the one hand and the revised Rescorla-Wagner model of Van Hamme and Wasserman (1994) and revised SOP model of Dickinson and Burke (1996) on the other hand, all of which could explain the results of Experiments 1-3. The design of Experiment 4 is depicted in the lower panel of Table 2. Based on the finding of Denniston et al. (1996; also see Miller \& Matute, 1997) that biologically significant cues (i.e., cues that already elicit appreciable responding) are more resistant to cue competition than are more neutral cues, we sought to maximize sensitivity to cue competition by preventing the target stimulus $(\mathrm{Y})$ from 
becoming biologically significant during training through our use of a sensory preconditioning procedure ( Brogden, 1939). In Phase 1, subjects in the overshadowing groups (OV-A and OVCxt) were presented with $A X$ followed by the surrogate US (S; $A X \rightarrow S$ ). As in Experiments 1-3, CS A was more salient than CS X; therefore, we anticipated overshadowing in these groups as was previously observed. Subjects in the control groups (Con-A and Con-Cxt) received $X \rightarrow S$ pairings. In Phase 2, all subjects received blocking training in which CSs $X$ and $Y$ were compounded and followed by $S(X Y \rightarrow S)$. In Phase 3, subjects in Groups OV-A and Con-A received massive extinction of the overshadowing stimulus $(A)$, whereas subjects in Groups OVCxt and Con-Cxt received equivalent exposure to the experimental context. Finally, prior to testing the target CS $(\mathrm{Y})$, all subjects received $\mathrm{S} \rightarrow \mathrm{US}$ pairings in order to provide a motivational basis for responding at test.

Based on the view that the comparator hypothesis applies to retrieval rather than response generation, we anticipated that the overshadowed CS $(X)$ would fail to block conditioned responding to $Y$ in Group OV-Cxt for the same reason that CS X was expected to fail to support strong behavioral control. If CS $X$ fails to support robust responding (due to strong $X-A$ and strong A-US associations), then it should also be unable to down-modulate responding to other stimuli. However, extinction of the overshadowing CS (A) following Phase 2 blocking training should allow $C S X$ to support strong behavioral control (as a consequence of the weakening of the $\mathrm{X}-\mathrm{A}$ and $\mathrm{A}-\mathrm{US}$ associations). Hence, the strong response potential of $\mathrm{CS} X$ should allow it to 'retrospectively' block conditioned responding to CS Y. Therefore, we expected to observe weak conditioned suppression to CS Y in Group OV-A relative to Group OV-Cxt. (A more precise statement of the mechanisms producing this effect are provided in the General Discussion.) In contrast, the revised Rescorla-Wagner (Van Hamme \& Wasserman, 1994) and revised SOP ( Dickinson \& Burke, 1996) models fail to anticipate weak responding to CS Y in Group OV-A. This follows from the lack of an A-Y within compound association. That is, extinction of CS A should result in retrospective revaluation of CS X, but that should have no effect on the associative status of CS Y.

We chose to omit the stimulus specificity control conditions in the present experiment because the previous three experiments all demonstrated that the increased responding to CS X (or CS Z) was the result of extinction of CS A (i.e., it was associative). Notably, in the present experiment, we expected that extinction of CS A would enhance responding to CS $X$ (which was not assessed in this experiment), thereby attenuating responding to CS Y. The omission of the specificity control groups leaves open the possibility that the anticipated attenuation of responding to CS Y would be merely the result of generalized extinction from CS A to CS Y. However, this possibility seems implausible given that we did not observe such an effect in the previous three studies which used the same stimuli and training parameters (e.g., Groups Con$A$ and Con-B in Experiments 1 and 2). 


\section{Method}

\section{Subjects and apparatus}

The subjects were 24 male (247-399 g) and 24 female (206-291 g), 80-120-day-old, naive, Sprague-Dawley descended rats from our SUNY-Binghamton breeding colony. Subjects were maintained and housed as in Experiments 1-3 and were randomly assigned to one of four groups (ns=12), counterbalanced for sex. The apparatus was the same as in Experiment 3 , except that CS A was the high-frequency complex tone, CS X was the white noise, and CS Y was the click train for all subjects. As in Experiment 3, CS A was more salient than CS X and CSs $X$ and $Y$ were of approximately equal salience.

\section{Procedure}

On Day 1, all subjects were acclimated to the experimental context as in Experiment 1. Phase 1 training (Days 2 and 3 ) for all subjects consisted of four daily $A X \rightarrow S$ pairings (at 10, 20, 37, and 50 min into each 60-min session), expect for Groups Con-A and Con-Cxt which received $X \rightarrow S$ rather than $A X \rightarrow S$ pairings. Stimulus $S$ served as the surrogate for the US and was presented for $5 \mathrm{~s}$ immediately following termination of $A X$ or $X$. BY training, analogous to $A X$ training, was omitted because previous studies had found that fewer stimuli usually result in less within-group variance, and Experiments 1-3 had already demonstrated stimulus specificity. Phase 2 training (Day 4) consisted of blocking training, during which all subjects received $4 \mathrm{XY} \rightarrow \mathrm{S}$ pairings (at $10,20,37$, and 50 min into the 60-min session). Phase 3 training (Days 5-8) consisted of 54 daily nonreinforced presentations of $A$ (Groups OV-A and Con-A) or equivalent context exposure (Groups OV-Cxt and Con-Cxt) during each daily 135-min session. For Groups OV-A and Con-A, stimulus presentations occurred on average every $2.5 \mathrm{~min}$ (range 1-4 min). Phase 4 training (Day 9) for all subjects consisted of four $S \rightarrow U S$ pairings (at 10, 20, 37, and 50 min into the 60-min session), with the US being presented immediately following termination of $S$. Lick tubes were absent during Phases 1-4. Reacclimation (Days 10 and 11) and Testing (Day 12) were the same as in Experiment 1, except that subjects were tested for conditioned suppression to $Y$, rather than $X$. That is, the mean time to complete 5 cumulative seconds of drinking in the presence of $Y$ served as the dependent variable. No subject required greater than $60 \mathrm{~s}$ to complete the five cumulative seconds of drinking in the absence of the test stimulus.

\section{Results and discussion}

Overshadowed stimulus $X$ failed to block conditioned responding to CS Y in Group OV-Cxt. However, extinction of the overshadowing stimulus $(A)$ following $X Y$-US blocking treatment attenuated conditioned suppression to $Y$ in Group OV-A. The mean times to complete five cumulative seconds of drinking in the presence of $Y$ are depicted in Fig. 3. As can be seen in the figure, Group OV-Cxt showed strong behavioral control by CS Y, whereas Group OV-A, which received extinction of CS A following blocking training, showed weak conditioned 
responding to $\mathrm{CS} Y$, indicative of retrospective blocking by CS X. These findings were confirmed with the following statistical analyses.

Analysis of the (log) times to complete five cumulative seconds of drinking in the presence of the test stimulus, CS Y was accomplished with a 2×2 ANOVA with Training Treatment (OV or Con) and Extinction ( $\mathrm{A}$ or $\mathrm{Cxt}$ ) as factors.1 This ANOVA revealed main effects of Training Treatment, $F(1,44)=18.66, p<.001$, and Extinction, $F(1,44)=20.84, p<.001$, as well as an interaction, $F(1,44)=14.10, p<.001$. Planned comparisons using the error term from the latter ANOVA were then conducted to identify the source of this interaction. These comparisons revealed that Group OV-Cxt suppressed more to $Y$ than did Group Con-Cxt, $F(1,44)=32.60$, $p<.001$, a finding indicative of the overshadowed stimulus failing to block conditioned responding to $Y$ in Group OV-Cxt. Additionally, Group OV-A suppressed less to $Y$ than did Group OV-Cxt, $F(1,44)=34.61, p<.001$, indicating that massive extinction of CS A in Group OVA allowed the overshadowed stimulus to retrospectively block conditioned responding to $\mathrm{Y}$.

In summary, Experiment 4 demonstrated that an overshadowed stimulus failed to block conditioned responding to a stimulus following blocking training. However, extinguishing the overshadowing stimulus following blocking treatment induced blocking. The robust responding to the blocked stimulus (Y) by Group OV-Cxt is consistent with Williams' (1996) findings. Recall that Williams found that a blocked stimulus failed to block acquisition of behavioral control by a novel stimulus. Similarly, here we found that an overshadowed stimulus failed to block acquisition of conditioned responding to a novel stimulus. Moreover, we additionally determined that massive posttraining extinction of the overshadowing CS allowed the overshadowed CS to retrospectively block conditioned responding to the novel stimulus. To the extent that processes in first-order preparations parallel processes in second-order preparations, this last observation calls into question prior interpretations of cue competition in terms of an associative acquisition deficit and additionally challenges acquisition-focused models of retrospective revaluation.

\section{GENERAL DISCUSSION}

This series of experiments examined whether the response deficit observed following cue competition treatment results primarily from an associative acquisition deficit or a performance deficit. Experiment 1 demonstrated both the basic overshadowing effect and that massive posttraining extinction of the overshadowing stimulus produces stimulus-specific recovery from overshadowing. Experiment 2 found that an overshadowed stimulus failed to support secondorder conditioning, unless massive posttraining extinction of the overshadowing stimulus was provided prior to second-order conditioning training. When the overshadowing stimulus was extinguished prior to second-order conditioning training, the previously overshadowed stimulus supported strong second-order responding. Experiment 3 replicated the results of Experiment 1, that is, stimulus specific recovery from overshadowing, using a sensory preconditioning procedure. Finally, Experiment 4 found that an overshadowed stimulus failed to block acquisition of behavioral control by a previously neutral stimulus, but that massive extinction of the overshadowing stimulus following the blocking treatment allowed the overshadowed stimulus to 'retrospectively block' responding to the previously neutral cue. 
Experiments 1 and 2 served as conceptual replications of the studies by Williams (1996) and Rauhut et al. (1999). Rauhut et al. found that massive extinction of a blocking stimulus or overshadowing stimulus failed to attenuate the blocking or overshadowing deficits, an effect that they claimed reflects an associative acquisition deficit rather than a performance deficit (as posited by comparator theory). Their findings are problematic not only for comparator theory, but also potentially so for the recently revised associative acquisition models that were designed to account for empirical retrospective revaluation (i.e., Dickinson \& Burke, 1996; Van Hamme \& Wasserman, 1994). Additionally, Rauhut et al.'s failure to observe recovery from overshadowing and blocking stands in contrast to numerous successful demonstrations of recovery from cue competition from a variety of laboratories, using different procedures and species (e.g., Best et al., 1985; Blaisdell and Blaisdell; Cole et al., 1995; Dickinson \& Charnock, 1985; Hallam et al., 1990; Kasprow et al., 1987; Kaufman \& Bolles, 1981; Lysle \& Fowler, 1985; Matzel et al., 1985; Miller et al., 1991; Schachtman et al., 1987; Shanks, 1985; Wasserman \& Berglan, 1998). Toward reconciling the discrepancy between Rauhut et al.'s findings and present and prior successful demonstrations of recovery from cue competition, we suggest that Rauhut et al.'s failure to observe recovery from overshadowing and blocking likely stems from procedural differences, specifically, their choice of stimuli (see Discussion of the present Experiment 1).

Another finding that both Williams (1996) and Rauhut et al. (1999, Experiment 2) viewed as problematic for comparator theory is the observed failure of a blocked or overshadowed stimulus to block acquisition of conditioned responding to a previously neutral stimulus. Additionally, Rauhut et al. found that a blocked or overshadowed stimulus failed to support second-order conditioning. The present Experiment 2 examined this last effect by providing subjects with overshadowing training followed by second-order conditioning in which the potential of the overshadowed stimulus to support second-order responding to a previously neutral cue was assessed. The overshadowed stimulus failed to support second-order responding unless subjects received massive extinction of the overshadowing CS prior to second-order conditioning training. This latter finding is consistent with comparator theory, but not traditional associative acquisition theory (e.g., Rescorla \& Wagner, 1972). That is, according to the comparator hypothesis, massive extinction of the overshadowing stimulus should weaken the A-US association and perhaps the $\mathrm{X}-\mathrm{A}$ association, resulting in a relatively weak indirectly activated US representation at test, thereby enhancing responding to the overshadowed stimulus (as demonstrated in the present Experiment 1). This attenuation of overshadowing should also allow the overshadowed stimulus to support second-order responding (as was observed in the present Experiment 2).

The results of Experiments 1 and 2 are consistent with a performance-deficit account of cue competition, but are also congruent with the recently revised associative acquisition-focused models designed to account for empirical retrospective revaluation (Dickinson \& Burke, 1996; Van Hamme \& Wasserman, 1994). According to these models, extinction of a competing cue results in an increment in the associative strength of the target stimulus despite the absence of the target CS during the extinction trials. Thus, both models predict an increment in associative strength to CS X, thereby allowing CS X to support both robust conditioned responding (as was observed in Experiments 1 and 3) and second-order conditioning (as was seen in Experiment 2). 
Experiments 3 and 4 were designed to differentiate the predictions of these revised associative acquisition models from the performance-deficit account of cue competition provided by comparator theory. In Experiment 4, subjects received overshadowing treatment followed by blocking treatment with the overshadowed CS serving as the blocking CS. Both revised SOP (Dickinson \& Burke, 1996) and revised Rescorla-Wagner ( Van Hamme \& Wasserman, 1994) predict no blocking of CS $Y$ by the overshadowed stimulus $X$, due to a weak X-US association. If CS A, the overshadowing stimulus, was extinguished prior to $X Y-U S$ blocking treatment, both models would anticipate strong blocking of $Y$ by $X$, due to an increment in associative strength to $C S X$ during extinction of $A$ increasing the expectation of the US (based on $X$ ) during $X Y \rightarrow U S$ blocking training (according to Van Hamme and Wasserman) or fewer elements of the US representation available for activation to the A1 state (according to Dickinson and Burke). However, providing extinction of CS A following blocking training should have no effect on responding to CS Y according to either model. This follows from the lack of an A-Y withincompound association. (Alternatively, one might posit that presentation of CS A activates a representation of $\mathrm{CS} X$, which in turn activates a representation of $\mathrm{CS} \mathrm{Y}$. If mediated activation of CS Y occurs, according to both models (Dickinson and Burke; Van Hamme and Wasserman) this should result in an increment in associative strength to CS Y, thereby leading to stronger responding to $C S Y$, rather than a retrospective blocking effect.) In the present Experiment 4, massive extinction of $\mathrm{CS} A$ following $X Y \rightarrow U S$ blocking treatment resulted in reduced responding to $\mathrm{CS} Y$, a result that is inconsistent with the predictions of the acquisition-focused models of retrospective revaluation.

The results of Williams' (1996, see also Rauhut et al., 1999, Experiment 2) study and our Experiment 4 (Group OV-Cxt) are seemingly inconsistent with the simple version of the comparator hypothesis originally presented by Miller and Matzel (1988), according to which both blocking and overshadowing result from a failure to express the target CS-US association as a result of strong target CS-comparator stimulus and comparator stimulus-US associations. The original comparator hypothesis assumed that the comparison of the strength of the US representation activated by the target CS and that activated by the comparator stimulus influenced response generation. Thus, the X-US association (in Williams' study and the present Experiment 4) should have been active and hence able to block acquisition of behavioral control by the blocked stimulus $\mathrm{Y}$, regardless of the behavioral control supported by CS X. Thus, the original comparator hypothesis predicts that Williams should have found blocking of a novel stimulus when it was trained in the presence of a previously blocked stimulus. To accommodate these findings, we have suggested that the comparator process not only modulates conditioned responding, but also the potential of a stimulus to modulate responding to other stimuli. Here we will provide a more detailed explanation of this process in terms of an extension of the comparator hypothesis (also see Blaisdell, Bristol, Gunther, \& Miller, 1998; Denniston et al., 2001) that posits that the modulatory potential of a comparator stimulus depends upon higherorder comparator stimuli. That is, the effectiveness of the comparator stimulus in modulating responding to its target CS is in part determined by its own comparator stimuli. When the comparator stimulus' own comparator stimulus indirectly activates a strong representation of the US, through strong higher-order comparator associations, the effectiveness of the target's comparator stimulus in modulating responding to the target CS will be reduced. 
A representation of the extended comparator hypothesis (as applied to Group OV-A of Experiment 4) is depicted in Fig. 4. According to the extended comparator hypothesis, the strength of the target CS-comparator stimulus association, Link 2, no longer reflects the absolute strength of the target CS-comparator stimulus association. Rather it reflects the effective strength of this association, which is potentially down-modulated by its own comparator process. That is, the strength of Link 2 (now called Link 2.1) is modulated by the comparator stimulus for Link 2.1, which is any and all other stimuli with strong associations to the target CS, excluding the first-order comparator stimulus and the US to prevent indeterminacy. As the strengths of Links 2.2 and 2.3 increase relative to the strength of Link 2.1, the activation of the effective first-order comparator stimulus will decrease. Conversely, as the strengths of Links 2.2 and 2.3 decrease, relative to the strength of Link 2.1, the activation of the effective first-order comparator stimulus will increase. The effectiveness of Link 3, now called Link 3.1, is similarly modulated by its own comparator process. In this case, the comparator stimulus for Link 3.1 is any and all stimuli with strong within-compound associations to the first-order comparator stimulus, excluding the target CS and the US. Strong Links 3.2 and 3.3 will down-modulate activation of the US representation by the first-order comparator stimulus, and the presence of weak Links 3.2 and 3.3 will permit activation of the US representation thereby facilitating the first-order comparator stimulus' effectiveness in modulating responding to the target CS. To summarize, the extended comparator hypothesis applies the comparator process to all relevant associations involved in the first-order comparator process, rather than only to the target CSUS association as was assumed in the original comparator hypothesis; thus, the extended comparator hypothesis is a more complete (i.e., general) statement of the original comparator hypothesis.

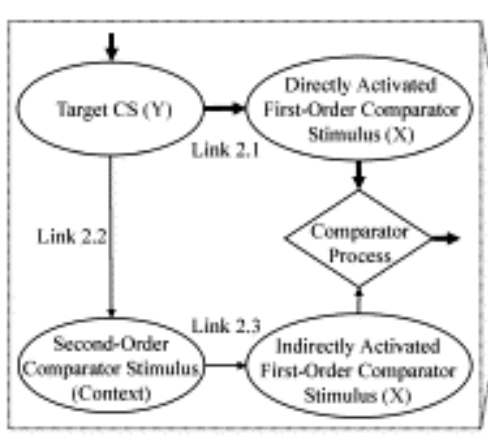

The Extended Comparator Hypothesis Applied to Experiment 4, Group $\mathrm{OV}=\mathrm{A}$

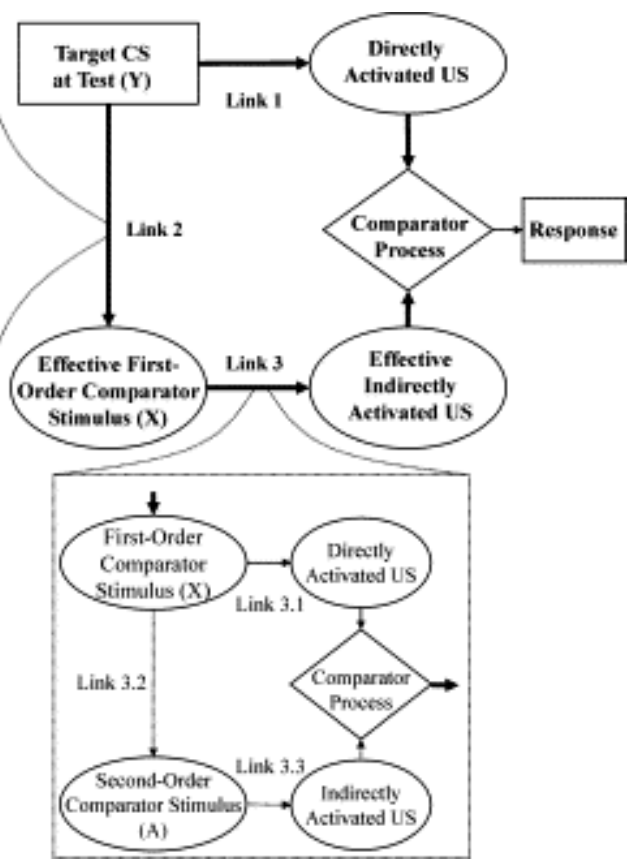


Fig. 4. The extended comparator hypothesis applied to the results of Experiment 4 (Group OVA). Although not depicted here, the model is to be applied simultaneously to all potential firstorder comparator stimuli. Comparator processes within dotted rectangles represent comparator processes for Links 2 and 3. Ovals depict stimulus representations; solid rectangles depict physical events; diamonds represent comparator processes. The target CS is CS Y; the firstorder comparator stimulus is CS X; the second-order comparator stimulus for Link 2 is the context; the second-order comparator stimulus for Link 3 is CS A. Boldface links indicate strong associations that lead to attenuated responding by Group OV-A.

Applied to Williams' (1996) study and the present Experiment 4 (Group OV-A), the extended comparator hypothesis provides a more complete explanation than the original comparator hypothesis of why an overshadowed or blocked stimulus $(X)$ fails to block acquisition of behavioral control by a previously neutral stimulus $(Y)$ unless extinction of the overshadowing or blocking stimulus (A) occurs following the Phase 2 blocking treatment (see Fig. 4). Following overshadowing (or blocking in the case of Williams) and subsequent blocking treatment, the effectiveness of the Y-US association is modulated by CS Y's comparator stimulus, CS X, which itself is subject to the comparator process. The effectiveness of the $Y-X$ association (Link 2.1 ) is modulated by the comparator stimulus for Link 2.1 , which would be any stimulus with a strong within-compound association to CS Y (other than CS X) which in this case would be the context. Following overshadowing (Phase 1) and blocking treatment (Phase 2), the strength of the $\mathrm{Y}-\mathrm{X}$ association should be strong relative to the $\mathrm{Y}$-context (Link 2.2) and context-X (Link 2.3) associations, due to the amount of time spent in the context in the absence of CSs $Y$ and $X$, respectively, and differences in salience between discrete and contextual stimuli. This should allow for strong activation of CS X as CS Y's first-order comparator stimulus. The effectiveness of the X-US association (Link 3.1) is similarly determined by the comparator stimulus for Link 3.1, which is any and all stimuli with strong within-compound associations to the first-order comparator stimulus. (Note that the comparator stimulus for Links 2.1 and 3.1 need not be the same.) As a result of overshadowing treatment, CS A would typically be a strong candidate comparator stimulus for CS X; however, the extinction of CS A provided in Phase 3 should have weakened both the $X-A$ and $A-U S$ associations (Links 3.2 and 3.3).[2] The presence of weak $X-A$ (Link 3.2) and A-US (Link 3.3) associations should have failed to down-modulate the $X-U S$ association (Link 3.1). That is, the relatively strong $X-U S$ association (Link 3.1) should have produced a robust indirect activation of the US. Thus, following overshadowing, subsequent blocking treatment, and extinction of CS A, presentation of CS Y should have strongly activated a representation of the first-order comparator stimulus $X$, and $X$ should have strongly activated a representation of the US, due to the comparator process for Link 3.1. This should have prevented CS Y from supporting strong conditioned responding at test.

Among contemporary models, the results of Experiment 4 (Group OV-A) uniquely support the extended comparator hypothesis. Subjects in Group OV-A received overshadowing treatment (Phase 1) followed by blocking treatment (Phase 2) and subsequent extinction of the overshadowing stimulus A (Phase 3). This training resulted in a retrospective blocking effect, in which responding to $\mathrm{CS} Y$ was attenuated relative to subjects that merely received exposure to the context during Phase 3 (Group OV-Cxt). According to the extended comparator hypothesis, the reduced blocking effect observed in Group OV-A is the result of the comparator process for 
Link 3.1 (see Fig. 4). Extinction of the overshadowing stimulus, $A$, resulted in a weakening of the A-US association (Link 3.3) and possibly the $X-A$ association (Link 3.2), thereby allowing CS X to activate a strong US representation. This now strong Link 3.1, combined with a previously strong Link 2.1, should allow CS Y to indirectly activate a strong US representation (mediated by CS X, which is Y's comparator stimulus) at test. This strong indirectly activated representation of the US should down-modulate responding to CS Y, a prediction supported by the results of Experiment 4 (Group OV-A). As previously stated, these results are not explicable in terms of the recently revised associative acquisition models of Dickinson and Burke (1996) and Van Hamme and Wasserman (1994) because CS A does not have a within-compound association to CS Y.

The present series of studies examined whether cue competition in general, and overshadowing in particular, result from an expression deficit rather than an acquisition deficit. The results of these experiments lend support to the extended comparator hypothesis, in which the effectiveness of a comparator stimulus is determined by higher-order comparator stimuli. When these higher-order comparator stimuli indirectly activate a strong representation of the first-order comparator stimulus (Link 2) or the US (Link 3), they presumably reduce the effectiveness of the first-order comparator stimulus in down-modulating responding to its target CS. Furthermore, posttraining deflation of the higher-order comparator stimuli should facilitate the first-order comparator stimulus in modulating responding to the target CS. Thus, an overshadowed or blocked stimulus should fail to block acquisition of behavioral control to a novel stimulus only when a strong comparator stimulus-US association is maintained following blocking training with the novel stimulus. Any treatment that weakens the effectiveness of the higher-order comparator stimuli (e.g., posttraining extinction of the higher-order comparator stimulus) should allow the first-order comparator cue to serve as a more effective blocking cue. The present data are congruent with this view.

\section{NOTES}

1. In each experiment, analysis of log times to complete the initial five cumulative seconds of drinking on the test day prior to the onset of the test stimulus was accomplished with a twofactor ANOVA. In Experiments 1-3, the factors were Training Treatment (OV or Con) and Extinction ( $A$ or $B$ ), whereas in Experiment 4, the factors were Training Treatment (OV or Con) and Extinction (A or Cxt). These analyses revealed no main effect of Training Treatment or Extinction, and no interaction, all $F s<1$ in Experiments $1-3$ and all $F s<2.27, p s>.13$ in Experiment 4, thereby demonstrating that there were no differences between groups in baseline drinking behavior prior to test stimulus presentation.

2. According to the extended comparator hypothesis, any and all stimuli present during training of a stimulus may serve as potential comparator stimuli, with the exception of the target stimulus (the test stimulus) and the US. That is, the comparator process is applied to all potential stimuli. For example, Fig. 4 portrays CS A as the comparator stimulus for Link 3. Presumably the context could also serve as a comparator stimulus for CS X, but its effect should be minimal (as depicted in Link 2). One might question whether CS A would remain the primary comparator 
stimulus for Link 3 following massive extinction treatment of CS A. That is, might the comparator stimulus for CS $X$ shift from CS A to CS Y? For the purpose of analyzing the response potential of CS $Y$, we omit CS $Y$ from serving as a comparator stimulus to prevent indeterminacy. However, if CS $X$ were the target stimulus, then CS Y might serve as a potential comparator stimulus for CS X. In this circumstance, the extended comparator hypothesis would anticipate more attenuated responding to CS X (due to the $\mathrm{Y}-\mathrm{US}$ association) than if the blocking treatment had not been provided. However, this was not of central interest in the present study.

\section{REFERENCES}

Best et al., 1985. M.R. Best, D.P. Dunn, J.D. Batson, C.L. Meachum and S.M. Nash , Extinguishing conditioned inhibition in flavour-aversion learning: Effects of repeated testing and extinction of the excitatory element. Quarterly Journal of Experimental Psychology 37B (1985), pp. 359-378.

Blaisdell et al., 1998. A.P. Blaisdell, A.S. Bristol, L.M. Gunther and R.R. Miller , Overshadowing and latent inhibition counteract each other: Support for the comparator hypothesis. Journal of Experimental Psychology: Animal Behavior Processes 24 (1998), pp. 335-351.

Blaisdell et al., 2001. A.P. Blaisdell, J.C. Denniston and R.R. Miller, Recovery from the overexpectation effect: Contrasting performance-focused and acquisition-focused models of retrospective revaluation. Animal Learning \& Behavior 29 (2001), pp. 367-380.

Blaisdell et al., 1999. A.P. Blaisdell, L.M. Gunther and R.R. Miller, Recovery from blocking through deflation of the block stimulus. Animal Learning \& Behavior 27 (1999), pp. 63-76.

Brogden, 1939. W.J. Brogden, Sensory preconditioning. Journal of Experimental Psychology 25 (1939), pp. 323-332.

Bush and Mostellar, 1955. R.R. Bush and F. Mostellar, Stochastic models for learning. , Wiley, New York (1955).

Cole et al., 1995. R.P. Cole, R.C. Barnet and R.R. Miller, Effect of relative stimulus validity: Learning or performance deficit?. Journal of Experimental Psychology: Animal Behavior Processes 21 (1995), pp. 293-303.

Denniston et al., 1996. J.C. Denniston, R.R. Miller and H. Matute, Biological significance as a determinant of cue competition. Psychological Science 7 (1996), pp. 325-331.

Denniston et al., 2001. J.C. Denniston, H.I. Savastano and R.R. Miller , The extended comparator hypothesis: Learning by contiguity, responding by relative strength. In: R.R. Mowrer and S.B. Klein, Editors, Handbook of contemporary learning theories, Lawrence Erlbaum Associates, Inc, Mahwah, NJ (2001), pp. 65-117. 
Dickinson and Burke, 1996. A. Dickinson and J. Burke, Within-compound associations mediate the retrospective revaluation of causality judgments. Quarterly Journal of Experimental Psychology 49B (1996), pp. 60-80.

Dickinson and Charnock, 1985. A. Dickinson and D.J. Charnock, Contingency effects with maintained instrumental reinforcement. Quarterly Journal of Experimental Psychology 37B (1985), pp. 397-416.

Gallistel and Gibbon, 2000. C.R. Gallistel and J. Gibbon , Time, rate, and conditioning. Psychological Review 107 (2000), pp. 289-344.

Hallam et al., 1990. S.C. Hallam, L.D. Matzel, J. Sloat and R.R. Miller, Excitation and inhibition as a function of posttraining extinction of the excitatory cue used in Pavlovian inhibition training. Learning and Motivation 21 (1990), pp. 59-84.

Holland, 1999. P.C. Holland, Overshadowing and blocking as acquisition deficits: No recovery after extinction of overshadowing or blocking cues. The Quarterly Journal of Experimental Psychology 52B (1999), pp. 307-334.

Kamin, 1968. L.J. Kamin , "Attention-like” processes in classical conditioning. In: M.R. Jones, Editor, Miami symposium on the prediction of behavior: Aversive stimulation, University of Miami Press, Miami, FL (1968), pp. 9-31.

Kasprow et al., 1987. W.J. Kasprow, T.R. Schachtman and R.R. Miller , The comparator hypothesis of conditioned response generation: Manifest conditioned excitation and inhibition as a function of relative excitatory associative strengths of CS and conditioning context at the time of testing. Journal of Experimental Psychology: Animal Behavior Processes 13 (1987), pp. 395406.

Kaufman and Bolles, 1981. M.A. Kaufman and R.C. Bolles , A nonassociative aspect of overshadowing. Bulletin of the Psychonomic Society 18 (1981), pp. 318-320.

Lysle and Fowler, 1985. D.T. Lysle and H. Fowler , Inhibition as a "slave" process: Deactivation of conditioned inhibition through extinction of conditioned excitation. Journal of Experimental Psychology: Animal Behavior Processes 11 (1985), pp. 71-94.

Mackintosh, 1975. N.J. Mackintosh , A theory of attention: Variations in the associability of stimuli with reinforcement. Psychological Review 82 (1975), pp. 276-298.

Matzel et al., 1985. L.D. Matzel, T.R. Schachtman and R.R. Miller, Recovery of an overshadowed association achieved by extinction of the overshadowing stimulus. Learning and Motivation 16 (1985), pp. 398-412.

Miller et al., 1992. R.R. Miller, R.C. Barnet and N.J. Grahame, Responding to a conditioned stimulus depends on the current associative status of other cues that were present during training of that specific stimulus. Journal of Experimental Psychology: Animal Behavior Processes 18 (1992), pp. 251-264. 
Miller et al., 1991. R.R. Miller, S.C. Hallam, J.Y. Hong and D.S. Dufore, Associative structure of differential inhibition: Implications for models of conditioned inhibition. Journal of Experimental Psychology: Animal Behavior Processes 17 (1991), pp. 141-150.

Miller and Matute, 1997. R.R. Miller and H. Matute, Biological significance in forward and backward blocking: Resolution of a discrepancy between animal conditioning and human causal judgment. Journal of Experimental Psychology: General 125 (1997), pp. 370-386.

Miller and Matzel, 1988. R.R. Miller and L.D. Matzel, The comparator hypothesis: A response rule for the expression of associations. In: G.H. Bower, Editor, The psychology of learning and motivation Vol. 22, Academic Press, San Diego, CA (1988), pp. 51-92. Abstract | View Record in Scopus | Cited By in Scopus (275)

Miller and Schachtman, 1985. R.R. Miller and T.R. Schachtman, Conditioning context as an associative baseline: Implications for response generation and the nature of conditioned inhibition. In: R.R. Miller and N.E. Spear, Editors, Information processing in animals: Conditioned inhibition, Erlbaum, Hillsdale, NJ (1985), pp. 51-88.

Pavlov, 1927. I.P. Pavlov, Conditioned reflexes., Oxford University Press, London (1927).

Pearce, 1987. J.M. Pearce , A model for stimulus generalization in Pavlovian conditioning. Psychological Review 94 (1987), pp. 61-73.

Pearce and Hall, 1980. J.M. Pearce and G. Hall , A model for Pavlovian conditioning: Variations in the effectiveness of conditioned but not unconditioned stimuli. Psychological Review 87 (1980), pp. 332-352.

Rauhut et al., 1999. A.S. Rauhut, J.E. McPhee and J.J.B. Ayres, Blocked and overshadowed stimuli are weakened in their ability to serve as blockers and second-order reinforcers in Pavlovian fear conditioning. Journal of Experimental Psychology: Animal Behavior Processes 25 (1999), pp. 45-67.

Rauhut et al., 2000. A.S. Rauhut, J.E. McPhee, N.T. DiPietro and J.J.B. Ayres, Conditioned inhibition training of the competing cue after compound conditioning does not reduce cue competition. Animal Learning \& Behavior 28 (2000), pp. 92-108.

Rescorla and Wagner, 1972. R.A. Rescorla and A.R. Wagner, A theory of Pavlovian conditioning: Variations in the effectiveness of reinforcement and nonreinforcement. In: A.H. Black and W.F. Prokasy, Editors, Classical conditioning II: Current research and theory, Appleton-Century-Crofts, New York (1972), pp. 64-99.

Schachtman et al., 1987. T.R. Schachtman, A.M. Brown, E. Gordon, D. Catterson and R.R. Miller, Mechanisms underlying retarded emergence of conditioned responding following inhibitory training: Evidence for the comparator hypothesis. Journal of Experimental Psychology: Animal Behavior Processes 13 (1987), pp. 310-322. 
Schachtman et al., 1992. T.R. Schachtman, W.J. Kasprow, R.C. Meyer, M.J. Bourne and J.A. Hart, Extinction of the overshadowing CS after overshadowing in conditioned taste aversion. Animal Learning \& Behavior 20 (1992), pp. 207-218.

Shanks, 1985. D.R. Shanks, Forward and backward blocking in human contingency judgment. Quarterly Journal of Experimental Psychology 37B (1985), pp. 1-21.

Speers et al., 1980. M.A. Speers, D.J. Gillan and R.A. Rescorla, Within-compound associations in a variety of compound conditioning procedures. Learning and Motivation 11 (1980), pp. 135149.

Van Hamme and Wasserman, 1994. L.J. Van Hamme and E.A. Wasserman, Cue competition in causality judgments: The role of nonpresentation of compound stimulus elements. Learning and Motivation 25 (1994), pp. 127-151.

Wagner, 1981. A.R. Wagner, SOP: A model of automatic memory processing in animal behavior. In: N.E. Spear and R.R. Miller, Editors, Information processing in animals: Memory mechanisms, Erlbaum, Hillsdale, NJ (1981), pp. 5-47.

Wasserman and Berglan, 1998. E.A. Wasserman and L.R. Berglan , Backward blocking and recovery from overshadowing in human causality judgment: The role of within-compound associations. The Quarterly Journal of Experimental Psychology 51B (1998), pp. 121-138.

Williams, 1996. B.A. Williams , Evidence that blocking is due to an associative deficit: Blocking history affects the degree of subsequent associative competition. Psychonomic Bulletin \& Review 3 (1996), pp. 71-74. 